\title{
MPS.BR Nível E - Uma Avaliação em Verde e Amarelo
}

\author{
Elaine Duarte Nunes ${ }^{1}$, Rosângela Silva ${ }^{1,2}$, Ana Regina Rocha ${ }^{2}$, Gleison Santos ${ }^{2}$ \\ ${ }^{1}$ Value Team Brasil \\ Rua da Candelária, 60, $10^{\circ}$ andar, CEP 20091-020, Rio de Janeiro, Brasil \\ ${ }^{2}$ COPPE/UFRJ - Universidade Federal do Rio de Janeiro \\ Caixa Postal 68511 - CEP 21945-970 - Rio de Janeiro, RJ \\ \{elaine.nunes, rosangela.pinto\} @valueteam.com.br \\ \{darocha, gleison\} acos.ufrj.br
}

\begin{abstract}
This articles describes an approach for software process deployment, with the achievement of MPS.BR (Brazilian Software Process Improvement) Level E Official Appraisal, after the organization experience of simultaneously obtaining the NBR ISO 9001:2000 certification and CMMI (Capability Maturity Model Integration) Level 2.
\end{abstract}

Resumo. Este artigo descreve uma abordagem para implantação de processos de software, com a realização de avaliação Nivel E do MPS.BR (Melhoria de Processo do Software Brasileiro,) após a experiência da organização na certificação NBR ISO 9001:2000 e a obtenção do Nível 2 do CMMI (Capability Maturity Model Integration) de forma simultânea.

\section{Introdução}

Qualidade é o fator crítico para o sucesso do negócio de software, como em qualquer área. E o caminho seguro para o aumento da qualidade é implantar e melhorar o processo de desenvolvimento de software.

O modelo de referência MPS.BR (Softex, 2005) é uma iniciativa brasileira, sob a coordenação da Sociedade SOFTEX, que visa a Melhoria de Processo do Software Brasileiro em todas as regiões do país, com foco nas pequenas e médias empresas (PMEs), a um custo acessível. O modelo possui como base a ISO/IEC 12207 (ISO/IEC, 2000), a ISO/IEC 15504 (ISO/IEC, 2003) e é compatível com o modelo CMMI (Chrissis et al., 2003). Os objetivos e práticas das áreas de processo do CMMI (níveis 2, 3, 4 e 5) foram distribuídos em 7 níveis de maturidade do modelo MPS.BR: G (Parcialmente Gerenciado), F (Gerenciado), E (Parcialmente definido), D (Largamente Definido), C (Definido), B (Gerenciado Quantitativamente) e A (Em Otimização).

A Relacional obteve a certificação ISO 9001:2000 (ISO, 2000) e o Nível 2 do CMMI após avaliações realizadas em março de 2005 (Duarte et al., 2005). A partir deste marco, sempre visando a melhoria contínua, uma nova meta foi estabelecida: a avaliação Nível E do MPS.BR. Este nível pode ser considerado como o estágio inicial do Nível 3 do CMMI e é composto dos seguintes processos: Treinamento, Definição do Processo Organizacional, Avaliação e Melhoria do Processo Organizacional e Adaptação do Processo para a Gerência 
de Projeto, além de todos os processos presentes no Nível G. Para cada um desses processos do MPS.BR existe um processo na ISO 12207 e uma área de processo CMMI correspondente. No CMMI, tem-se respectivamente, as seguintes áreas de processo: Organizational Training, Organizational Process Definition, Organizational Process Focus e Integrated Project Management.

Este artigo está organizado em cinco seções. Na primeira seção é apresentada a introdução; na seção 2, a empresa; na seção 3, o foco é o projeto para obtenção do Nível E do MPS.BR; na seção 4, apresentamos como resultado obtido, a avaliação MPS.BR e, finalmente, na seção 5, apresentamos algumas conclusões e ressaltamos as perspectivas futuras da organização em seu objetivo de melhoria contínua dos processos.

\section{A Empresa}

A Relacional Consultoria Ltda foi criada no ano de 1989 e no início a empresa concentrou-se no segmento de comércio exterior. Logo, com a necessidade de manter e expandir a empresa, a atuação foi sendo diversificada. No final dos anos 90 houve uma expansão da carteira de clientes acompanhada pela expansão física e de dimensão da empresa. Hoje, além da sede no Rio de Janeiro, há escritórios em São Paulo (desde 1999), Belo Horizonte (desde 2001), São José dos Campos (desde 2001) e Brasília (desde 2004). Atualmente a Relacional possui 508 funcionários. Dos 377 funcionários do Rio de Janeiro, há 347 profissionais trabalhando no desenvolvimento de software. A Relacional tem como foco desenvolvimento, manutenção (Service Level Agreement) e terceirização (Outsourcing) em consultoria de sistemas (Fábrica de Software-Projetos e/ou Programas) em Web, Cliente-Servidor, DataWarehouse, Business Intelligence, Help Desk, treinamento em tecnologia da informação, suporte técnico e administração de redes.

\section{Projeto para a Avaliação Nível E MPS.BR}

Assim como na obtenção do Nível 2 do CMMI, a Relacional contou, durante a preparação para o Nível E do MPS.BR, com a consultoria da COPPE/UFRJ. A primeira atividade neste projeto foi realizar um mapeamento para identificar os "gaps" entre os processos existentes na empresa, as áreas de processo do CMMI Nível 2 e os processos dos níveis G, F e E do MPS.BR.

\subsection{Mapeamento CMMI X MPS.BR}

A realização deste mapeamento se fez necessária, pois algumas subpráticas do CMMI (não obrigatórias, pelo modelo) foram elevadas ao status de resultados esperados (obrigatórios) no MPS.BR, além de existirem resultados que não estão presentes no CMMI, por pertencerem a ISO/IEC 12207 / ISO/IEC 15504. Um exemplo está no processo Gerência de Configuração, onde o MPS.BR exige a existência de uma rotina de backup. No caso da Relacional, devido à implantação de processos visando a certificação ISO 9001:2000, que cobre todas as áreas da empresa, os procedimentos da área de Suporte já estavam documentados e, por consequiência, o procedimento de backup. Sendo assim, não foi necessária a criação de um novo processo ou artefato, pois a evidência para o resultado esperado no MPS.BR já existia. Isso aconteceu em várias outras situações por já haver os 
processos documentados para a ISO 9001 ou por já haver processos implantados que ultrapassavam as exigências do Nível 2 do CMMI.

\subsection{Definição do Processo Organizacional}

O propósito desse processo é estabelecer e manter um conjunto de ativos dos processos organizacionais, usável e aplicável às necessidades de negócio da organização.

Antes da avaliação CMMI Nível 2, a Relacional já utilizava um processo de desenvolvimento, chamado MDS - Metodologia de Desenvolvimento de Sistemas, difundido na organização desde 1991, que estava em sua quarta versão. Mesmo não sendo exigido neste Nível do CMMI, a Relacional manteve este processo, adaptando-o às exigências do modelo, lançando a versão 5 da MDS. Para atender ao processo Definição do Processo Organizacional, do MPS.BR, foi documentado o que na prática já estava definido/implementado. Isto sempre ocorrerá quando a empresa assumir que é primordial ter um processo definido desde o inicio da implantação de um programa de melhoria de processo, quer seja para o CMMI ou para o MPS.BR.

\subsection{Adaptação do Processo}

O propósito desse processo é estabelecer e gerenciar o projeto e envolver os interessados relevantes de acordo com o processo definido e integrado que é adaptado do conjunto de processos-padrão da organização.

Este processo também já estava implantado na empresa. A abordagem de implantação de processos utilizada implicava em ter-se um processo padrão integrando os processos definidos para as áreas de processo CMMI e apoiar o desenvolvimento dos projetos utilizando o Ambiente TABA desenvolvido na COPPE/UFRJ (Rocha et al., 2005). Com o uso deste ambiente a primeira atividade de um projeto é adaptar o processo padrão da organização para o projeto específico, através da ferramenta AdaptPro (Villela et al., 2004), e em seguida planejar o desenvolvimento de acordo com o processo planejado. Novamente, portanto, o que se fez, para atender a este processo MPS.BR foi documentar o que de fato já existia na empresa.

\subsection{Avaliação e Melhoria do Processo Organizacional}

O propósito desse processo é determinar o quanto os processos-padrão da organização contribuem para a organização alcançar os objetivos de negócio e ajudam a organização a planejar e implementar melhorias contínuas nos processos com base no entendimento de seus pontos fortes e fracos.

Logo após a avaliação CMMI Nível 2 foi lançada a versão 5.1 da MDS, contemplando todas as melhorias identificadas na avaliação, assim como as sugestões coletadas ao longo a utilização da versão 5 pelos líderes. Com a implantação deste processo, as formas de coleta para a melhoria do processo ficaram mais definidas.

A Figura 1 mostra onde as oportunidades de melhorias são coletadas: através dos resultados das avaliações externas CMMI, ISO e MPS.BR; através da avaliação do líder/equipe realizada ao final de cada fase da MDS; e através do repositório de métricas, que 
poderá identificar tendências. Essas oportunidades servem como entrada para este processo (Foco do Processo Organizacional), retornando ao ciclo inicial.

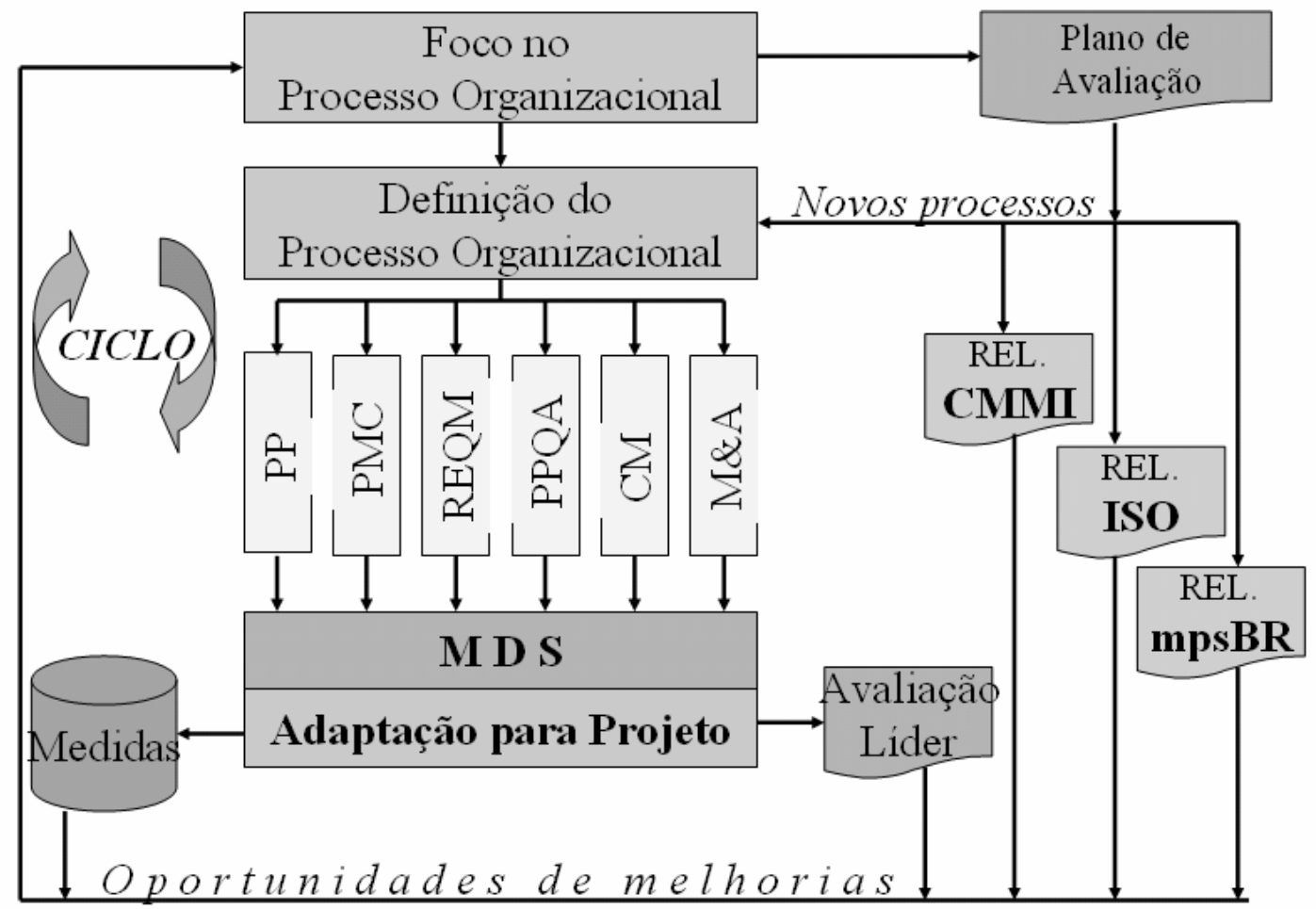

Treinamento Organizacional (a base para todos os processos)

Figura 1. Ciclo de Melhorias nos Processos

\subsection{Treinamento}

O propósito desse processo é prover a organização e os projetos com profissionais que possuam os conhecimentos e as habilidades necessárias para executar as suas funções de forma efetiva.

Para definição deste processo MPS.BR foi considerado o processo definido e implantado para a certificação ISO, sob a responsabilidade da área de Recursos Humanos, ajustado às exigências do MPS.BR. A empresa coleta as necessidades de treinamento através da avaliação dos funcionários, que ocorre anualmente. A partir dessas necessidades, é estabelecido o Programa de Treinamento e os treinamentos são oferecidos no decorrer do ano. A eficácia dos treinamentos é avaliada na próxima avaliação, quando, então, são novamente coletadas as necessidades. O ciclo se completa: Avaliação dos funcionários $\rightarrow$ Necessidade $\rightarrow$ Programa de Treinamento $\rightarrow$ Treinamento $\rightarrow$ Avaliação dos funcionários.

\subsection{Preparação para a Avaliação}

Toda a documentação dos processos foi elaborada em apenas um mês. Este curto tempo somente fi possível devido ao nível dos processos já implantados na organização para o CMMI Nível 2 e a ISO 9001, restando apenas ser formalizada a definição dos novos 
processos exigidos para o Nível E do MPS.BR e que de fato já estavam implantados na empresa.

O acompanhamento contínuo dos processos e projetos após a realização da avaliação SCAMPI (SEI, 2001) foi um fator decisivo para a rapidez na preparação para o Nível E. Um outro fator a ser destacado nesta etapa de preparação para a avaliação Nível E foi a seriedade de todos os colaboradores da empresa. Toda a experiência no planejamento, preparação e infra-estrutura efetuados para a avaliação CMMI foram transferidos para a avaliação MPS.BR:

- Planejamento - Elaboração do cronograma das atividades, envolvimento de outras áreas para suprir a infra-estrutura da avaliação, como administrativo e suporte;

- Preparação - Convocação dos participantes, publicação do cronograma, comunicação aos funcionários sobre o Kickoff da avaliação, confecção de cartazes;

- Infra-estrutura - Materiais e equipamentos necessários, sala de entrevistas, sala do Time Appraisal, logística de lanche/almoço, criação da chave Visitante, acesso de leitura ao diretório dos projetos avaliados.

\section{Resultados Obtidos e Lições Aprendidas}

Como resultado do projeto e lições aprendidas, descreveremos a própria avaliação MPS.BR. A equipe que avaliou o MPS.BR Nível E na Relacional contou com os seguintes avaliadores credenciados pela Sociedade SOFTEX: Cristina Filipak Machado (Curitiba) - como avaliadora-líder; Adriana Silveira de Souza (Goiânia), Alexandre Vasconcelos (Recife), Ana Guerra (Campinas), Claudia Welter (Porto Alegre) e Francisco Vasconcelos (Manaus) como avaliadores-adjuntos; além de Rosangela Pinto Silva - avaliadora representante da empresa.

A avaliação MPS.BR seguiu o mesmo molde do SCAMPI: abertura do evento com o avaliador-líder e o patrocinador; cumprimento do cronograma das entrevistas; verificação das evidências (artefatos dos projetos); apuração do resultado; apresentação preliminar dos resultados aos entrevistados; comunicação do resultado ao patrocinador e finalizando com a comunicação do resultado à empresa.

A Relacional foi a primeira empresa aprovada numa avaliação oficial do modelo MPS.BR no Nível E - Parcialmente Definido. O resultado foi divulgado no dia 29 de setembro de 2005, na sede da empresa no Rio de Janeiro.

Esta avaliação foi um marco. A Relacional realmente foi avaliada no Brasil, por uma equipe que reuniu avaliadores de norte a sul do país. Profissionais experientes em engenharia de software, com uma visão crítica, fundamental para uma avaliação de processos de software. A avaliação da Relacional foi uma das 5 avaliações-piloto MPS.BR realizadas em 2005 e a de mais alto nível MPS.BR.

A partir das informações coletadas nessas avaliações, haverá uma revisão do modelo e a definição final do processo e método de avaliação MPS.BR, previstas para publicação em maio 2006. Finalmente, cabe observar que esta avaliação foi uma importante ocasião de 
aprendizado, onde todos os envolvidos saíram ganhando: a equipe de avaliadores, a Relacional, o modelo MPS.BR e, principalmente, o Brasil.

Além disso, é importante destacar a percepção dos colaboradores da Relacional após a participação nos projetos de melhoria e nas avaliações do CMMI e do MPS.BR. A seguir, são reproduzidos três depoimentos que comprovam isto:

- Lauro Santanna - Líder de Projeto: "A implantação de um bom processo agrega segurança, confiabilidade, conhecimento e minimização de erros à execução de projetos, garantindo aos produtos gerados um padrão mínimo de qualidade. Além de que a formalização de atividades - mesmo que antes executadas (muitas vezes de forma quase que intuitiva) - traz embutida além de boas práticas, a inevitável discussão e análise crítica dessas mesmas atividades. Promovendo a conscientização da relação entre atividades e objetivos e, consequentemente, uma melhoria significativa. Penso que o CMMI é importante para claramente nortear os objetivos pretendidos quando da implementação de um processo, indicando um caminho a ser seguido. Sendo a certificação a "conseqüência natural" de uma maturidade na utilização desse processo, indicando formalmente que os objetivos desejados estão sendo alcançados e, também, possíveis fraquezas a serem melhoradas. E impulsionando a equipe a almejar novos objetivos para aprimorar o processo".

- Alexandre Losada - Líder de projeto: "O processo de software visa a qualidade do produto final, gerando métricas, possibilitando o alcance de prazos e custos adequados, gerando registros de todas as fases do projeto e criando um modo de trabalho que possa ser constantemente avaliado na tentativa de atingir um modelo ideal, aumentando o grau de competitividade da empresa. A adoção do modelo CMMI está se tornando uma exigência e um passaporte para comercialização internacional de produtos e soluções de Software. A indústria brasileira de Software necessita estar apta a demonstrar a maturidade de seus processos para ter livre acesso e competir em pé de igualdade nos mercados internacionais. A adesão à metodologia CMMI deve ser visando, primeiramente, à melhoria dos processos de produção e conseqüente redução de custos, prazos de entrega, retrabalhos etc. Hoje estamos cientes de que a certificação CMMI também é vital para cumprir a meta de atender o mercado externo e melhorar a posição no mercado interno."

- Rusemberg Maia - Membro do Grupo de Gerência de Configuração: 'É uma demonstração do grau de importância dado pela empresa ao profissionalismo de sua "mão de obra"; é uma demonstração do quanto a Relacional acredita que o forte de "uma organização" (ou empresa) é a sua organização; é uma comprovação que todos esforços da empresa são convergentes e que o rumo é o mesmo, tanto para CMMI quanto para MPS.BR ou para outro tipo de avaliação que venham a inventar. Em relação à avaliação MPS.BR, verifiquei que é bem mais criteriosa que o CMMI, oferecendo-nos um nível maior de dificuldade ao atendimento das metas. A vantagem é que seus resultados estão alinhados aos interesses do CMMI." 


\section{Conclusões e Perspectivas Futuras}

Em janeiro de 2006, a Relacional Consultoria foi incorporada à Value Team, do internacional e qualificado grupo Value Partners. O grupo, fundado em 1993, em Milão, Itália, rapidamente se tornou o terceiro maior grupo de consultoria da Europa. A equipe da Relacional se junta a consultores de 20 países, que atendem a grandes empresas em diversas partes do mundo. Dentro desse contexto, as perspectivas futuras passam a ser da Value Team, que seguirá trilhando o mesmo caminho de qualidade iniciado pela Relacional.

Quando o Nível 2 do CMMI foi alcançado pela empresa, a Guia Geral do MPS.BR ainda não tinha sido lançada. O caminho natural é galgar o CMMI, através do MPS.BR. E é este caminho a ser seguido pela Value Team. Antes da avaliação CMMI Nível 3, haverá a avaliação MPS.BR Nível C, assim como prevemos uma avaliação MPS.BR Nível A, antes da avaliação CMMI Nível 5. Adotaremos as avaliações formais MPS.BR como uma préavaliação, porém com resultado válido em todo território brasileiro.

O resultado das avaliações é com certeza a maior maturidade da capacidade de desenvolver software com qualidade, bem como ter-se uma equipe confiante, que acredita no processo e que cada vez mais entende a importância da melhoria contínua, conforme os depoimentos dos colaboradores evidenciam.

\section{Agradecimentos}

A Sociedade Softex, aos membros do Grupo de Processos (Angela Abreu, Carlos Quintanilha e Rusemberg Maia), aos líderes dos projetos avaliados (Lígia Castro, Glayne Sampaio, Grace Pereira e Williams Monteiro), à equipe de avaliadores MPS.BR, a equipe de desenvolvimento da Estação TABA e a todos os funcionários da Relacional que participaram da avaliação.

\section{Referências}

Chrissis, M. B., Konrad, M, Shrum, S. (2003) "CMMI: Guidelines for Process Integration and Product Improvement", Addison-Wesley.

Duarte, E., Silva R., Natali, A., Rocha, A., Santos, G. (2005) "Uma Abordagem para Implantação de Processos de Software com ISO 9001 e CMMI". IV Simpósio Brasileiro de Qualidade de Software, SBQS'05, Porto Alegre, RS, Brasil.

ISO 9001:2000 - Quality management systems - Requirements, (2000)

ISO/IEC 12207:2000 - Information technology -software process life cycle, (2000).

ISO/IEC, ISO/IEC TR 15504-2003: Information Technology - Software Process Assessment, Parts 1-9, International Organization for Standardization and the International Electrotechnical Commission, Geneva, Switzerland.

Rocha, A. R., Montoni, M., Santos, G., Montoni, M., Mafra, S., Figueiredo, S., Bessa, A., Mian, P. (2005) "Estação TABA: Uma Infra-estrutura para Implantação do Modelo de Referência para Melhoria de Processo de Software”, IV SBQS, Porto Alegre, Brasil. 
SEI, (2001), "Standard CMMI® Appraisal Method for Process Improvement (SCAMPISM), Version 1.1: Method Definition Document", disponível em http://www.sei.cmu.edu, verificado em Maio/2006.

SOFTEX, (2005), "MPS.BR - Melhoria de Processo de Software: Guia Geral”, Abril.

Villela, K., Santos, G., Montoni, M., Berger, P., Figueiredo, S, M., Mafra, S, N., Rocha, A, R, C., Travassos, G, H. (2004) "Definição de Processos em Ambientes de Desenvolvimento de Software Orientados a Organização", III Simpósio Brasileiro de Qualidade de Software, Brasília-DF. 